\title{
Energy Cooperation in Energy Harvesting Two-Way Communications
}

\author{
Berk Gurakan $^{1}$, Omur Ozel ${ }^{1}$, Jing Yang ${ }^{2}$, and Sennur Ulukus ${ }^{1}$ \\ ${ }^{1}$ Department of Electrical and Computer Engineering, University of Maryland, College Park, MD 20742 \\ ${ }^{2}$ Department of Electrical Engineering, University of Arkansas, Fayetteville, AR 72701
}

\begin{abstract}
In this paper, we investigate a two-way communication channel where users can harvest energy from nature and energy can be transferred in one-way from one of the users to the other. Energy required for data transmission is randomly harvested by the users throughout the communication duration and users have unlimited batteries to store energy for future use. In addition, there is a separate wireless energy transfer unit that facilitates energy transfer only in one-way and with efficiency $\alpha$. We study the energy cooperation made possible by wireless energy transfer in the two-way channel. Assuming that both users know the energy arrivals in advance, we find jointly optimal offline energy management policies that maximize the sum throughput of the users. We show that this problem is a convex optimization problem, and find the solution by a generalized two-dimensional directional water-filling algorithm which transfers energy from one user to another while maintaining that the energy is allocated in the time dimension optimally. Optimal solution equalizes the energy levels as much as possible both among users and among slots, permitted by causality constraints of the energy arrivals and one-way energy transfer.
\end{abstract}

\section{INTRODUCTION}

The conventional two-way communication channel introduced in [1] consists of two users sending messages to each other simultaneously over the same physical medium using their own resources. In such a communication scenario, even though the users send independent data using their independent resources, they may implicitly cooperate by feeding back some information about the signal they received in previous channel uses. For the memoryless additive Gaussian two-way channel, the overall two-way channel decomposes into two independent one-way additive Gaussian channels and users achieve their single-user capacities simultaneously [2]. Another dimension of cooperation arises when energy transfer over the physical medium is possible [3], [4]: Energy may be abundant in one user in which case the loss incurred by transferring it to the other user may be less than the gain it yields for the other user. It is this cooperation that we wish to explore in this paper which we call energy cooperation.

We consider a two-way communication channel composed of two users powered by energy harvesting devices. These devices can harvest energy from the surrounding environment as in solar cells, water mills or mechanical vibration absorption devices, etc. In particular, users communicate by using energy harvested from the environment. We model this scenario

This work was supported by NSF Grants CNS 09-64632, CCF 09-64645, CCF 10-18185 and CNS 11-47811.

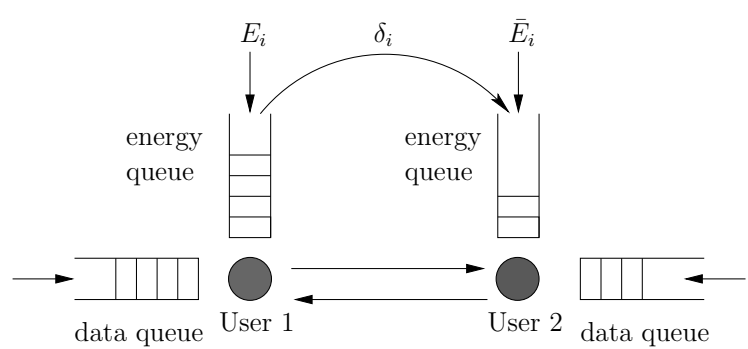

Fig. 1. Two-way communication model with energy cooperation.

as two users having independent exogenous energy arrival processes that recharge their batteries. Additionally, we assume that one-way energy transfer is possible: The first user can transmit a portion of its energy to the second user through a separate wireless energy transfer unit subject to a delay and attenuation as shown in Fig. 1. This is motivated by practical applications such as RFID systems in which the passive reader is powered by the energy reflected on it by the sender [5]. Neglecting the other energy expenditures such as computation and sensing, we focus on the communication performances of the users under the energy harvesting and one-way energy transfer constraints. We study the optimization of transmission policies of the users in this two-way communication channel with energy harvesting and energy transfer. Assuming that the users know the realizations of the energy arrival processes in advance, as in the existing literature [6]-[19], we find sum throughput maximizing optimal offline transmission and energy transfer policies of the users.

Offline throughput maximization for an energy harvesting transmitter has been the subject of the recent literature [6][19]. In [6], transmission completion time minimization problem for an energy harvesting transmitter is solved. In [7], throughput maximization problem by a deadline is solved for an energy harvesting transmitter in a static channel. [8][15] extend the throughput maximization problem and its solution to fading, broadcast, multiple access, interference and relay channels. Extensions of this problem to battery imperfections are considered in [16], [17] and processing costs are considered in [18], [19]. In [3], we extended the two-hop cooperative network problem [14], [15] to a setup where source to relay wireless energy transfer is allowed. Recently, in [20] two-way communication channel with energy exchange with a fixed amount of energy in the system is 
considered where the channels in each direction are noiseless and the energy transfer is lossless. Unlike [20], in our setup the channels are noisy and the energy transfer is lossy.

Although energy transfer via RF energy harvesting may be inefficient due to path-losses over the wireless channel [21], more efficient wireless energy transfer methods based on magnetic coils exist [22], [23]. We, therefore, base our model on the possibility of efficient wireless energy transfer and extend our previous work in [3] to a two-way communication channel with energy harvesting users and one-way energy transfer. We formulate the offline sum throughput maximization problem with energy transfer subject to energy causality at both users. We show that this problem is a convex optimization problem, and give the solution via a generalized two-dimensional directional water-filling algorithm. The energy causality constraints take a new form when energy transfer is possible: energy can flow in time from the past to the future for each user and from the first user to the second at each time. We utilize right permeable taps for energy flow through time for a single user and down permeable taps for energy transfer between users. In the optimal solution, due to the concavity of the throughput in powers, energy must be spread as equally as possible both among users and among time slots, subject to the causality constraints of energy arrivals and one-way energy transfer. We extend the sum rate maximization formulation in this paper to the entire capacity region in [4].

\section{System Model and Problem Formulation}

We consider a two-way communication channel consisting of two users as shown in Fig. 1. The two queues at the nodes are the data and energy queues with unlimited sizes. The energies that arrive at the nodes are saved in the corresponding energy queues. The data queues of both users always carry some data packets. In addition, energy expenditure is only due to data transmission; any other energy costs, e.g., processing, are not considered. There is a separate one-way wireless energy transfer unit at the first user, which transfers energy from the first user to the second user.

We assume a memoryless Gaussian two-way channel as described in [2]. The channel inputs and outputs at the first and second user are $x_{1}, x_{2}$ and $y_{1}, y_{2}$, respectively. Input-output relations are $y_{1}=x_{1}+x_{2}+n_{1}$ and $y_{2}=x_{1}+x_{2}+n_{2}$ where $n_{1}$ and $n_{2}$ are independent Gaussian additive noises with zeromean and unit variances. We assume that the time is slotted and there are a total of $T$ equal length slots assumed to be of unit length. At times $t=1, \ldots, T$, user 1 harvests energy with amounts $E_{1}, E_{2}, \ldots, E_{T}$ and user 2 harvests energy with amounts $\bar{E}_{1}, \bar{E}_{2}, \ldots, \bar{E}_{T}$. One-way energy transfer efficiency is $\alpha$, where $0 \leq \alpha \leq 1$ : When the first user transfers $\delta_{i}$ amount of energy to the second user, $\delta_{i}$ amount of energy exits the first user's energy queue and $\alpha \delta_{i}$ amount of energy enters the second user's energy queue in the same slot. Power policy of user 1 is the sequences $P_{i}$ and $\delta_{i}$; power policy of user 2 is the sequence $\bar{P}_{i}$.

For both users, the energy that has not arrived yet cannot be used for data transmission or energy transfer, yielding the following energy causality constraints:

$$
\begin{aligned}
& \sum_{i=1}^{k} P_{i} \leq \sum_{i=1}^{k}\left(E_{i}-\delta_{i}\right), \quad k=1, \ldots, T \\
& \sum_{i=1}^{k} \bar{P}_{i} \leq \sum_{i=1}^{k}\left(\bar{E}_{i}+\alpha \delta_{i}\right), \quad k=1, \ldots, T
\end{aligned}
$$

For the Gaussian two-way channel with individual power constraints $P_{1}$ and $P_{2}$, rate pairs $\left(R_{1}, R_{2}\right)$ with $R_{1} \leq$ $\frac{1}{2} \log \left(1+P_{1}\right), R_{2} \leq \frac{1}{2} \log \left(1+P_{2}\right)$ are achievable [2]. We formulate the sum throughput maximization problem with energy transfer subject to energy causality at both users as:

$$
\begin{aligned}
\max _{\bar{P}_{i}, P_{i}, \delta_{i}} & \sum_{i=1}^{T} \frac{1}{2} \log \left(1+P_{i}\right)+\frac{1}{2} \log \left(1+\bar{P}_{i}\right) \\
\text { s.t. } & \sum_{i=1}^{k} P_{i} \leq \sum_{i=1}^{k}\left(E_{i}-\delta_{i}\right), \quad k=1, \ldots, T \\
& \sum_{i=1}^{k} \bar{P}_{i} \leq \sum_{i=1}^{k}\left(\bar{E}_{i}+\alpha \delta_{i}\right), \quad k=1, \ldots, T \\
& \sum_{i=1}^{k} \delta_{i} \leq \sum_{i=1}^{k} E_{i}, \quad k=1, \ldots, T
\end{aligned}
$$

The problem in (3) is a convex optimization problem as the objective function is concave and the feasible set is a convex set [24]. We write the Lagrangian function for (3) as

$$
\begin{aligned}
\mathcal{L}= & -\sum_{i=1}^{T} \log \left(1+P_{i}\right)+\log \left(1+\bar{P}_{i}\right) \\
& +\sum_{k=1}^{T} \mu_{k}\left(\sum_{i=1}^{k} P_{i}-\left(E_{i}-\delta_{i}\right)\right) \\
& +\sum_{k=1}^{T} \eta_{k}\left(\sum_{i=1}^{k} \bar{P}_{i}-\left(\bar{E}_{i}+\alpha \delta_{i}\right)\right)-\sum_{k=1}^{T} \rho_{k} \delta_{k}
\end{aligned}
$$

The Lagrange multiplier $\rho_{k}$ is due to the constraint that $\delta_{k} \geq 0$. Note that the same constraints apply for $P_{i}$ and $\bar{P}_{i}$; however, $P_{i}$ and $\bar{P}_{i}$ are always nonzero in the optimal policy, therefore we exclude those Lagrange multipliers. Similarly, we exclude the constraints $\sum_{i=1}^{k} \delta_{i} \leq \sum_{i=1}^{k} E_{i}$ in the Lagrangian function as these constraints can never be satisfied with equality in the optimal policy ${ }^{1}$. The KKT conditions for this problem are:

$$
\begin{aligned}
\frac{-1}{1+P_{i}}+\sum_{k=i}^{T} \mu_{k} & =0, \quad i=1, \ldots, T \\
\frac{-1}{1+\bar{P}_{i}}+\sum_{k=i}^{T} \eta_{k} & =0, \quad i=1, \ldots, T \\
\sum_{k=i}^{T} \mu_{k}-\alpha \sum_{k=i}^{T} \eta_{k}-\rho_{i} & =0, \quad i=1, \ldots, T
\end{aligned}
$$

\footnotetext{
${ }^{1}$ If user 1 transfers all of its energy at any slot then its instantaneous rate will be zero. Since the objective function is concave in $P_{i}$ and $\bar{P}_{i}$ the optimal solution can never have $P_{i}=0$.
} 
with the additional complementary slackness conditions as:

$$
\begin{aligned}
\mu_{k}\left(\sum_{i=1}^{k} P_{i}-\left(E_{i}-\delta_{i}\right)\right) & =0, \quad \forall k \\
\eta_{k}\left(\sum_{i=1}^{k} \bar{P}_{i}-\left(\bar{E}_{i}+\alpha \delta_{i}\right)\right) & =0, \quad \forall k \\
\rho_{k} \delta_{k} & =0, \quad \forall k
\end{aligned}
$$

From (5), (6) and (7) we get:

$$
\begin{aligned}
P_{i} & =\frac{1}{\sum_{k=i}^{T} \mu_{k}}-1, \quad i=1, \ldots, T \\
\bar{P}_{i} & =\frac{1}{\sum_{k=i}^{T} \eta_{k}}-1, \quad i=1, \ldots, T \\
\rho_{i} & =\sum_{k=i}^{T} \mu_{k}-\alpha \sum_{k=i}^{T} \eta_{k}, \quad i=1, \ldots, T
\end{aligned}
$$

Next, we find the optimal power allocation policies $P_{i}$ and $\bar{P}_{i}$ and the optimal energy transfer profile $\delta_{i}$ for all values of $\alpha$.

\section{Optimal Power Allocation AND Energy TRANSFER PROFILE}

We start by noting that for a given energy transfer profile $\delta_{1}, \ldots, \delta_{T}$ the optimization problem can be separated between the users and the optimal power profiles $P_{i}^{*}$ and $\bar{P}_{i}^{*}$ can be found by the directional water-filling algorithm described in [8] or the geometric approach described in [6] applied to the energy arrival profiles $E_{i}-\delta_{i}$ and $\bar{E}_{i}+\alpha \delta_{i}$ for $i=1, \ldots, T$. This means that both users' power allocation profiles are nondecreasing and only increasing whenever they exhaust all of the energy in their respective batteries [6], [8].

Lemma 1 The optimal power sequences $P_{i}^{*}$ and $\bar{P}_{i}^{*}$ are monotonically increasing sequences: $P_{i+1}^{*} \geq P_{i}^{*}, \bar{P}_{i+1}^{*} \geq \bar{P}_{i}^{*}$. Moreover if for some $l, \sum_{i=1}^{l} P_{i}^{*}<\sum_{i=1}^{l} E_{i}-\delta_{i}^{*}$ then $P_{l}^{*}=$ $P_{l+1}^{*}$. Similarly, if for some $j, \sum_{i=1}^{j} \bar{P}_{i}^{*}<\sum_{i=1}^{j} \bar{E}_{i}+\alpha \delta_{i}^{*}$ then $\bar{P}_{j}^{*}=\bar{P}_{j+1}^{*}$.

Next, we provide the necessary optimality condition for energy transfer in the following lemma.

Lemma 2 For the optimal power sequences $P_{i}^{*}, \bar{P}_{i}^{*}$ and energy transfer sequence $\delta_{i}^{*}$, if $\delta_{i}^{*} \neq 0$ for any slot $i$ then,

$$
\frac{1+P_{i}^{*}}{1+\bar{P}_{i}^{*}}=\frac{1}{\alpha}
$$

Proof: From (11)-(13) we have

$$
\frac{1+P_{i}^{*}}{1+\bar{P}_{i}^{*}}=\frac{\sum_{k=i}^{T} \eta_{k}}{\alpha \sum_{k=i}^{T} \eta_{k}+\rho_{i}}
$$

If there is a non-zero energy transfer, $\delta_{i} \neq 0$, we have from (10), $\rho_{i}=0$. Therefore, (14) must be satisfied if $\delta_{i} \neq 0$.

Lemmas 1 and 2 will be useful for finding the optimal power allocation and energy transfer profiles. Now we divide the problem into two cases, namely $\alpha=1$ and $\alpha<1$.

\section{A. Energy transfer efficiency $\alpha=1$}

We consider the case of $\alpha=1$ to gain insight on the optimal solution to this problem. We start by restating (11) and (12) in the following way,

$$
\begin{aligned}
& P_{i}^{*}=\nu_{i}-1, \quad i=1, \ldots, T \\
& \bar{P}_{i}^{*}=\bar{\nu}_{i}-1, \quad i=1, \ldots, T
\end{aligned}
$$

where the water levels $\nu_{i}$ and $\bar{\nu}_{i}$ in slot $i$ are given by

$$
\begin{aligned}
\nu_{i} & =\frac{1}{\sum_{k=i}^{T} \mu_{k}}, \quad i=1, \ldots, T \\
\bar{\nu}_{i} & =\frac{1}{\sum_{k=i}^{T} \eta_{k}}, \quad i=1, \ldots, T
\end{aligned}
$$

When $\alpha=1$, from Lemma 2 we have $\bar{\nu}_{i}=\nu_{i}$ whenever there is an energy transfer. If some energy is transferred through users, the power levels at that slot must equalize. In view of Lemma 1, energy has to be allocated in time and user dimensions together. This calls for a two-dimensional directional water-filling algorithm where energy is allowed to flow in two dimensions, from left to right (in time) and from up to down (among users). We utilize right permeable taps to account for energy which will be used in the future and down permeable taps to account for energy transfers between users.

We consider the two slot system shown in Fig. 2. Whenever water flows through a tap (horizontal or vertical), water levels must equalize among those slots. Water can only flow from left to right and from up to down. In this example, $E_{1}$ is large enough so that levels of the first two slots of user 1 and first slot of user 2 are equalized in the optimal solution. Since $\bar{E}_{2}$ is large, there is no need for energy transfer at the second slot therefore no water flows through that tap.

The two dimensions of the water flow (i.e., in time and among users) in the resulting water-filling system are coupled. Therefore, finding the optimal water levels is challenging as it is not known beforehand which taps will be open or closed in the optimal solution. While an exhaustive search over the ordering of opening the taps may be proposed, we provide a concise algorithm to find the optimal solution. We let each tap (right/down permeable) have a meter measuring the water that has already passed through it. If a tap meter has a positive value, that tap becomes bidirectional and will allow water flow in both directions. This allows us to recall any energy that is sent to future slots or transferred to the other user suboptimally. First, we fill energy into the slots with all taps closed. Then, we open only the right permeable taps and perform directional water-filling for both users individually [8]. Each right permeable tap measures the total amount of energy moving through it. If a tap meter has a positive value, that tap becomes bidirectional in the following step. After this step, each user has increasing power/water levels.

Then, we open the down taps one by one in a backward fashion. If water flows down through a tap, the amount is measured by the meter. Water levels in the slots connected by the bidirectional taps and the down tap are equalized as much as possible. This implies that water may flow to the left (past) 

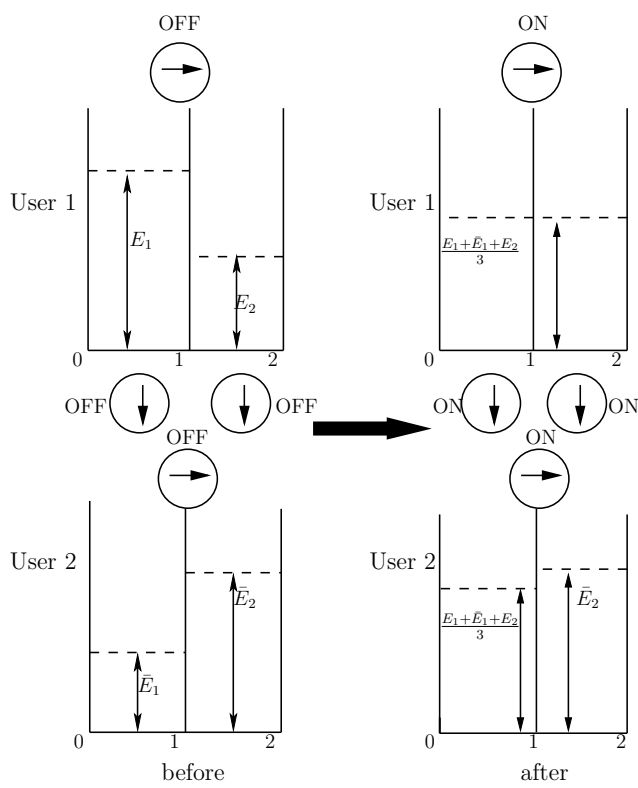

Fig. 2. Two-dimensional directional water-filling with right/down permeable taps for a two slot system.

through bidirectional taps in order to keep the water level even. If this happens, the amount of water moving back through the tap is subtracted from the meter. If a meter becomes zero, the tap becomes right-permeable again, and no water is allowed to flow left through it. If a down tap meter becomes positive, the down tap becomes bidirectional (up and down) in the remaining steps.

An example run of the algorithm is given in Fig. 3. Initially we open the right permeable taps and the water levels are equalized. Then, we open the down permeable taps. In the second slot there is no need for energy transfer because $\frac{E_{1}+E_{2}}{2}<\bar{E}_{2}$. In the first slot there will be some non-zero energy transfer since $\frac{E_{1}+E_{2}}{2}>\bar{E}_{1}$, and some water flows through the first down permeable tap. Since user 1's right permeable tap has a positive meter at that point, some water is allowed to flow from right to left thereby equalizing the water levels of user 1's first and second slots and user 2's first slot.

The optimality of this algorithm is verified by checking the KKT optimality conditions through (11)-(13) with $\alpha=1$. Since the algorithm only terminates whenever $\nu_{i} \leq \bar{\nu}_{i}, \forall i$ we have $\rho_{i}=\frac{1}{\nu_{i}}-\frac{1}{\bar{\nu}_{i}} \geq 0, \forall i$. We will find $\eta_{i}, \mu_{i}$ using (18) and (19). By construction of the algorithm, the power levels are always non-decreasing and equalize whenever some water flows across a directional tap. We define $\eta_{i}=\frac{1}{\bar{\nu}_{i}}-\frac{1}{\bar{\nu}_{i+1}}$ and $\mu_{i}=\frac{1}{\nu_{i}}-\frac{1}{\nu_{i+1}}, \forall i$. Since power levels are non-decreasing, we have $\mu_{i}, \eta_{i} \geq 0, \forall i$. This means that we can find unique $\eta_{i}, \mu_{i}, \rho_{i} \geq 0$ that satisfy the KKT optimality conditions therefore showing that the algorithm is optimal.

\section{B. Energy transfer efficiency $\alpha<1$}

For $\alpha<1$, we obtain the solution via a generalized twodimensional directional water-filling algorithm. To that end, we define $\tilde{P}_{i}=\frac{\bar{P}_{i}}{\alpha}$ and formulate the optimization problem in terms of $P_{i}, \tilde{P}_{i}$ and $\delta_{i}$ as follows:

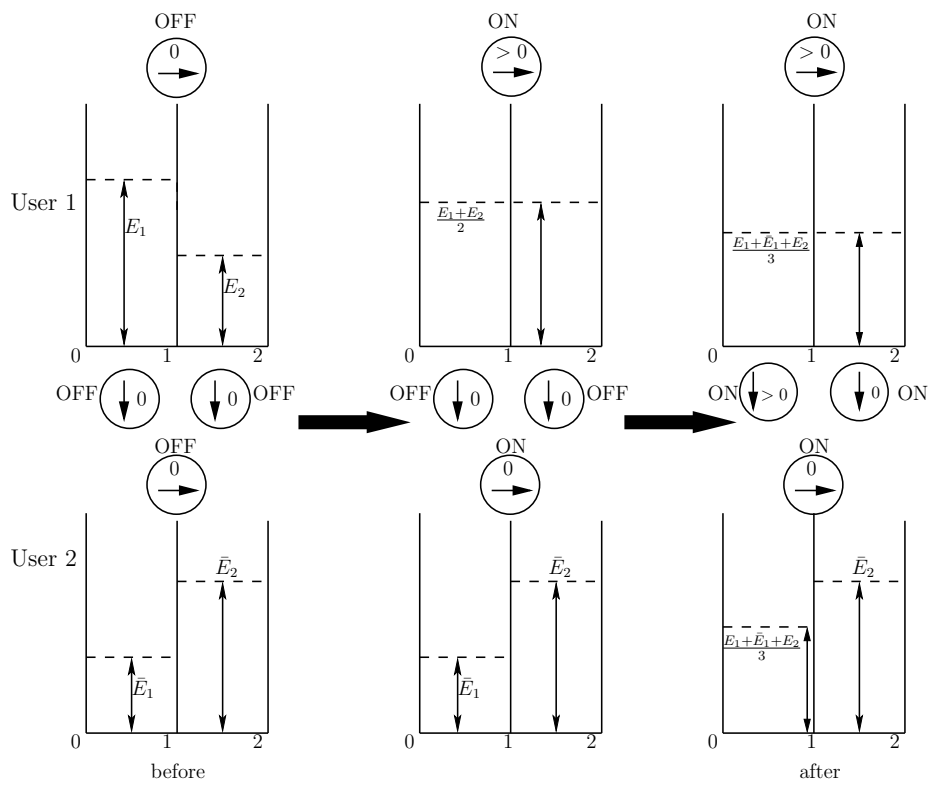

Fig. 3. Two-dimensional directional water-filling with right/down permeable meter taps for a two slot system: an example run of the algorithm.

$$
\begin{aligned}
\max _{\tilde{P}_{i}, P_{i}, \delta_{i}} & \sum_{i=1}^{T} \frac{1}{2} \log \left(1+P_{i}\right)+\frac{1}{2} \log \left(1+\alpha \tilde{P}_{i}\right) \\
\text { s.t. } & \sum_{i=1}^{k} P_{i} \leq \sum_{i=1}^{k}\left(E_{i}-\delta_{i}\right), \quad k=1, \ldots, T \\
& \sum_{i=1}^{k} \tilde{P}_{i} \leq \sum_{i=1}^{k}\left(\frac{\bar{E}_{i}}{\alpha}+\delta_{i}\right), \quad k=1, \ldots, T \\
& \sum_{i=1}^{k} \delta_{i} \leq \sum_{i=1}^{k} E_{i}, \quad k=1, \ldots, T
\end{aligned}
$$

Following the approach in Section II, the solution is

$$
\begin{aligned}
& P_{i}^{*}=\nu_{i}-1, \quad i=1, \ldots, T \\
& \tilde{P}_{i}^{*}=\tilde{\nu}_{i}-\frac{1}{\alpha}, \quad i=1, \ldots, T
\end{aligned}
$$

where $\nu_{i}$ and $\tilde{\nu}_{i}$ are the water levels for this new problem. We observe from (21) and (22) that the second user's bottom level is $\frac{1}{\alpha}$ while the first user's bottom level is 1 . In addition, the energy arrivals for the second user need to be scaled by $\frac{1}{\alpha}$. To find the optimal power levels, we make these two modifications and run the two-dimensional directional waterfilling algorithm on the modified system. This yields $\nu_{i}$ and $\tilde{\nu}_{i}$. We use (21), (22) and $\bar{P}_{i}=\alpha \tilde{P}_{i}$ to find the final power levels. The required modifications are explained in Fig. 4.

\section{NUMERICAL EXAMPLE}

In this section, we provide a numerical example over 3 slots to illustrate our algorithm. We assume that user 1 harvests $\mathbf{E}=[0,12,0] \mathrm{mJ}$ and user 2 harvests $\overline{\mathbf{E}}=[6,6,0] \mathrm{mJ}$ and $\alpha=1 . T_{1 i}, T_{2 i}$ denote the horizontal taps of the first and second users connecting the $i$ th and $i+1$ st slots, $Q_{i}$ denotes the $i$ th vertical tap. The optimal solution is $\mathbf{P}=[0,4.8,4.8]$ and $\overline{\mathbf{P}}=[4.8,4.8,4.8]$, which is obtained by spreading the energy 


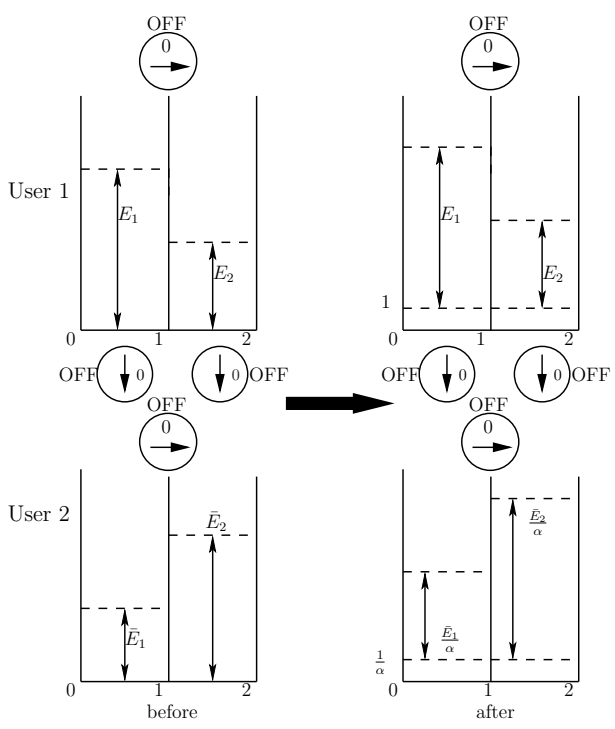

Fig. 4. The required modification to the initial water levels for $\alpha<1$.

as equally as possible in two dimensions among the users and time slots, subject to energy causality. In order to show why spreading the energy in two dimensions is challenging, we next consider two sub-optimal orderings of tap openings.

Assume that we open the horizontal taps first and keep the vertical taps closed. This yields the transient water levels $\mathbf{P}=[0,6,6]$ and $\overline{\mathbf{P}}=[4,4,4]$. Now, if we open the vertical taps, water is transferred in the second and third slots and the balanced final levels are $\mathbf{P}=[0,5,5]$ and $\overline{\mathbf{P}}=[4,5,5]$. This profile is not optimal since the second user changes its power level when the battery is non-empty, violating Lemma 1.

Now, assume that we open the vertical taps first and keep the horizontal taps closed. Energy is transferred in the second slot and the new transient water levels will be $\mathbf{P}=[0,9,0]$ and $\overline{\mathbf{P}}=[6,9,0]$. Then, when we open the horizontal taps, we have $\mathbf{P}=[0,4.5,4.5]$ and $\overline{\mathbf{P}}=[5,5,5]$. This profile is not optimal either, as after energy transfer, the first user's power level is less than the second user's power level, violating Lemma 2.

We now show how the two-dimensional directional waterfilling algorithm works. First, we open the horizontal taps to get $\mathbf{P}=[0,6,6]$ and $\overline{\mathbf{P}}=[4,4,4]$ with the tap meters reading $[0,6]$ and $[2,2]$. Recall that the taps with positive meter readings allow bidirectional energy transfer. Next, we open the vertical taps in a backward fashion. Once $Q_{3}$ is opened, water flows to the second user and since $T_{21}, T_{22}$ are bidirectional it starts to fill all the slots of the second user. A balance is established when $\mathbf{P}=[0,4.8,4.8]$ and $\overline{\mathbf{P}}=[4.8,4.8,4.8]$, which is the optimal solution.

\section{CONCLUSions}

In this paper, we addressed a two-way communication channel with energy harvesting rechargeable nodes. We formulated the offline sum throughput maximization problem with a oneway wireless energy transfer subject to energy causality at both nodes. We showed that this is a convex optimization problem. We gave the solution in terms of a generalized two- dimensional directional water-filling algorithm which utilizes metered right and down permeable taps for optimal energy flow among the users and in time.

\section{REFERENCES}

[1] C. E. Shannon, "Two-way communication channels," in Proc. 4th Berkeley Symp. Probability and Statistics, pp. 611-644, Univ. California Press, 1961.

[2] T. S. Han, "A general coding scheme for the two-way channel," IEEE Trans. Inform. Theory, vol. 30, pp. 35-44, January 1984.

[3] B. Gurakan, O. Ozel, J. Yang, and S. Ulukus, "Energy cooperation in energy harvesting wireless communications," in IEEE ISIT, July 2012.

[4] B. Gurakan, O. Ozel, J. Yang, and S. Ulukus, "Two-way and multipleaccess energy harvesting systems with energy cooperation," in 46th Asilomar Conf. Signals, Syst. and Comp., Nov. 2012.

[5] B. Glover and H. Bhatt, RFID Essentials. O'Reilly Media, 2006

[6] J. Yang and S. Ulukus, "Optimal packet scheduling in an energy harvesting communication system," IEEE Trans. Comm., vol. 60, pp. 220-230, January 2012.

[7] K. Tutuncuoglu and A. Yener, "Optimum transmission policies for battery limited energy harvesting nodes," IEEE Trans. Wireless Comm., vol. 11, pp. 1180-1189, March 2012.

[8] O. Ozel, K. Tutuncuoglu, J. Yang, S. Ulukus, and A. Yener, "Transmission with energy harvesting nodes in fading wireless channels: Optimal policies," IEEE Jour. on Selected Areas in Commun., vol. 29, pp. 17321743, September 2011.

[9] J. Yang, O. Ozel, and S. Ulukus, "Broadcasting with an energy harvesting rechargeable transmitter," IEEE Trans. Wireless Comm., vol. 11, pp. 571-583, February 2012.

[10] M. A. Antepli, E. Uysal-Biyikoglu, and H. Erkal, "Optimal packet scheduling on an energy harvesting broadcast link," IEEE Jour. on Selected Areas in Commun., vol. 29, pp. 1721-1731, September 2011.

[11] O. Ozel, J. Yang, and S. Ulukus, "Optimal broadcast scheduling for an energy harvesting rechargeable transmitter with a finite capacity battery," IEEE Trans. Wireless Comm., vol. 11, pp. 2193-2203, June 2012.

[12] J. Yang and S. Ulukus, "Optimal packet scheduling in a multiple access channel with rechargeable nodes," Journal of Communications and Networks, vol. 14, pp. 140-150, April 2012.

[13] K. Tutuncuoglu and A. Yener, "Sum-rate optimal power policies for energy harvesting transmitters in an interference channel," Journal of Communications and Networks, vol. 14, pp. 151-161, April 2012.

[14] C. Huang, R. Zhang, and S. Cui, "Throughput maximization for the Gaussian relay channel with energy harvesting constraints," IEEE Jour. on Selected Areas in Commun., to appear 2013.

[15] D. Gunduz and B. Devillers, "Two-hop communication with energy harvesting," in IEEE CAMSAP, December 2011

[16] B. Devillers and D. Gunduz, "A general framework for the optimization of energy harvesting communication systems with battery imperfections," Journal of Comm. and Netw., vol. 14, pp. 130-139, April 2012.

[17] K. Tutuncuoglu and A. Yener, "Communicating using an energy harvesting transmitter: Optimum policies under energy storage losses," IEEE Trans. Wireless Comm., submitted August 2012, available at [arXiv:1208.6273].

[18] O. Orhan, D. Gunduz, and E. Erkip, "Throughput maximization for an energy harvesting communication system with processing cost," in IEEE ITW, September 2012.

[19] J. Xu and R. Zhang, "Throughput optimal policies for energy harvesting wireless transmitters with non-ideal circuit power," IEEE Jour. on Selected Areas in Commun., submitted April 2012, available at [arXiv:1204.3818].

[20] P. Popovski and O. Simeone, "Two-way communication with energy exchange," in IEEE ITW, September 2012.

[21] P. Grover and A. Sahai, "Shannon meets Tesla: Wireless information and power transfer," in IEEE ISIT, July 2010.

[22] A. Kurs, A. Karalis, R. Moffatt, J. Joannopoulos, P. Fisher, and M. Soljacic, "Wireless power transfer via strongly coupled magnetic resonances," Science, vol. 317, pp. 83-86, Jul. 2007.

[23] A. K. J. Joannopoulos and M. Soljacic, "Efficient wireless non-radiative mid-range energy transfer," Annals of Physics, vol. 323, pp. $34-48$, Jan. 2008.

[24] S. Boyd and L. Vandenberghe, Convex Optimization. United Kingdom: Cambridge University Press, 2004. 